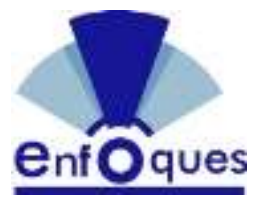

Enfoques. Revista de Investigación en Ciencias de la Administración http://doi.org/10.33996/revistaenfoques.v3i12.73

No. 12 | Volumen 3 | Octubre - Diciembre 2019 http://revistaenfoques.org ISSN: $2616-8219$ pp. $299-316$

\section{TÁCTICAS DE CIBERPOLÍTICA PARA POSICIONAR UN PARTIDO POLÍTICO EN BOLIVIA}

\author{
CYBERPOLITICAL TACTICS TO POSITION A POLITICAL PARTY \\ IN BOLIVIA
}

\author{
Mariana Belén Espinoza Arando
}

Artículo recibido julio 2019 | Arbitrado agosto - septiembre 2019 | Publicado 01 de octubre 2019

\section{Resumen}

Tácticas de ciberpolítica para incrementar el posicionamiento del partido político PDC (Partido Demócrata Cristiano) en la ciudad de Potosí, es la primera en el área de marketing político, dentro de la Universidad Privada Domingo Savio, tiene como estructura tres partes, en estas se analizan las bases teóricas y se establecen los métodos a utilizarse, por otro lado se tiene el diagnostico, determinado principalmente por las herramientas de investigación Encuesta, Entrevista y Observación. Por último, se tiene la propuesta realizada para poder subsanar el problema que se evidenció que es el bajo nivel de posicionamiento, para esta propuesta se tomó en cuenta un elemento nuevo en el marketing político que es la ciberpolítica. Es así que la finalidad del estudio, es resolver el problema acerca del bajo nivel de posicionamiento mediante las tácticas de ciberpolítica, teniendo en cuenta que en la actualidad todo lo referido al entorno del internet tiene mucho más relevancia y eficacia.

Palabras clave: Tácticas de ciberpolíticas; partidos políticos; marketing político

\begin{abstract}
Cyberpolitical tactics to increase the positioning of the political party PDC (Christian Democratic Party) in the city of Potosí, it is the first in the area of political marketing, within the Private University Domingo Savio, it has three parts as a structure, these are analyzed the theoretical bases and the methods to be used are established, on the other hand there is the diagnosis, determined mainly by the research tools Survey, Interview and Observation. Finally, there is the proposal made to correct the problem that was evidenced by the low level of positioning, for this proposal a new element was taken into account in the political marketing that is Cyberpolitics. Thus, the purpose of the study is to solve the problem about the low level of positioning through cyberpolitical tactics, taking into account that at present everything related to the internet environment is much more relevant and effective.
\end{abstract}

Key words: Cyberpolitical tactics; political parties; political marketing
Mariana Belén Espinoza Arando marbel--_--princes@hotmail.com Universidad Privada Domingo Savio, Bolivia

Ingeniera Comercia egresada Universidad Privada Domingo Savio. Experiencia en Inteligencia Comunicativa interpersonal v2.0. Impuestos Sobre Utilidades de las Empresas (IUE). Marketing Político. Marketing Digital 


\section{INTRODUCCIÓN}

El marketing en los tiempos actuales se utiliza como una herramienta imprescindible para el éxito de la empresa, institución u organización, teniendo en cuenta que para ofrecer cualquier tipo de bien, servicio o idea se requieren distintas tácticas con las que esta cuenta, y puestas en acción de manera científica o empírica como se conoce en el contexto socioeconómico-cultural, desde su creación es tomada como la bandera que encabeza la presentación y la innovación, para surgir de manera exitosa en cualquier tipo de mercado, así mismo en esta época como todo lo demás, ha evolucionado involucrándose de manera estrecha con la nueva tecnología.

Es por esto que han surgido nuevos y distintos tipos de marketing como: el marketing digital, marketing social, marketing verde, marketing político y muchos más, dedicándose cada uno de ellos a un mercado específico con herramientas similares y distintas a la vez, con el fin único de crear un valor hacia un producto, servicio o idea por parte de las personas, que en distintos casos se da un crecimiento económico o utilidades, pero en otros genera una concientización 0 apoyo significativo. Por lo tanto, para utilizar el marketing como tal, existen aspectos primordiales que se deben conocer, para saber o identificar el problema y que tácticas se debe utilizar para solucionar dicho problema.

Un aspecto de suma importancia que toda organización debe considerar es el posicionamiento, entendido como la posición que se ocupa en la mente de las personas, para llegar a ocupar esta posición las tareas, tácticas son varias, requiriendo de tiempo y una planificación ardua.

Es así que el posicionamiento es un aspecto tan importante, que al no conocer el producto, servicio o idea de la organización no se puede pensar en un crecimiento empresarial u organizacional. Por otro lado, se puede tomar al posicionamiento como fundamental dentro de distintos tipos del marketing, uno de ellos es el marketing político referido a llegar a la mente de las personas mediante un ideal, propuestas, marca, imagen, candidato, relaciones con la sociedad, objetivos, participación, militantes, entre otros, que genere, se puede hablar de un partido político conocido, es por esta razón que se toma al posicionamiento como el pilar férreo para seguir adelante en este mercado.

En consecuencia, para utilizar el marketing político, es importantes entrar en un mar inmenso de información sobre el nacimiento, desarrollo y actualidad de la política, hablando de su origen en el mundo, desde que el ser humano existe y realiza un proceso de socialización con otros de su especie, formando la familia que es el núcleo primordial que impulso al nacimiento de un gobernante. Con el trascurrir del tiempo se han adoptado dos corrientes políticas básicas; que son la izquierda, que como ideal utiliza la defensa de la igualdad económica y social, por otro lado, se tiene a la derecha, que acepta las diferencias económicas, sociales como algo inevitable y normal, es decir la defensa de la libertad individual, de la propiedad privada y del libre mercado.

Hoy en día la política utiliza ambas corrientes dentro de los partidos políticos, aquí es donde se emplean diferentes herramientas de marketing político, que muestra a su manera, cada una de estas corrientes, con representantes determinados y que hace que sus ciudadanos elijan a un gobernante y su forma de pensar.

En Bolivia se utiliza diferentes concepciones políticas. Haciendo un retroceso, se tuvieron varios gobiernos; 
pasando inclusive por dictaduras, estadías democráticas y actualmente se tiene un gobierno de izquierda (MAS-IPSP). Cerca de una elección presidencial para la gestión 2019, los partidos políticos y agrupaciones ciudadanas emplearán distintas estrategias de marketing político, actualmente existen nueve partidos políticos registrados en el Tribunal Supremo Electoral (TSE) a nivel nacional.

Conociendo este aspecto, se tiene al "Partido Demócrata Cristiano" fundado un 6 de febrero de 1954 como Partido Social Cristiano (PSC), y adoptó su actual nombre en un congreso del partido en noviembre de 1964 teniendo hoy 64 años de existencia. Se debe aclarar que se mantiene como un partido "tercerista", señalando una "tercera vía" entre el capitalismo y el socialismo, una vía que sería más humana y verdaderamente democrática. La presente organización fue fundada por Benjamín Miguel Harb, Javier Caballero y Emanuel Andrade. En la actualidad está a la cabeza de Lic. Luis Ayllon con más de 59.408 militantes registrados.

En consecuencia, el "Partido Demócrata Cristiano" también se establece en la ciudad de Potosí con todos los lineamientos y reglamentos ya cumplidos y busca generar de manera exitosa un posicionamiento político y social.

Contextualizando la problemática abordada en este estudio se tiene que de después de una evaluación interna, del partido político PDC en el municipio de Potosí, se determinó sé que encuentra en la etapa de introducción, en relación al ciclo de vida del partido político, debido a ello el partido político PDC no es muy conocido y es un riesgo que continúe en esta situación.

El posicionamiento, en uno de sus conceptos básicos guarda referencia a hacer sentir importante a los componentes del segmento objetivo, porque ellos son los que adquieren, aceptan o rechazan lo que se ofrece, en la política es igual, las personas buscan un partido político que se preocupe por ellos, que su interés no sea solo a nivel económico o personal quieren que un partido político establezca propuestas, ideas, etc. que los beneficie y les haga sentir importantes, el partido político PDC de acuerdo a la perspectiva actual tampoco cumple esto.

Es así, que cada parte, aspecto o característica por más mínima que sea podría generar la diferencia buena o mala, de un partido político con otro partido político, pero eso solo dependerá de la forma que una persona quiera ver las cosas, en el caso de la política es muy complicado, porque lo que a alguien le gusta a otro le puede desagradar.

Actualmente, para las personas lo que más importa de un partido político son las propuestas, a comparación de la imagen o el candidato, las propuestas son la base, la esencia de un partido político, sean estas propuestas económicas, de salud, de educación, de desarrollo, etc., es lo que beneficia $o$ perjudica a un país y sus habitantes, entonces, aunque se tenga el mejor candidato, una gran imagen, si las propuestas no son lo que quiere la sociedad, los partidos políticos son rechazados y por lo tanto no generan posicionamiento.

La marca de un partido político, engloba todo lo que es un partido, lo bueno, lo malo, el candidato las propuestas, su ideología, etc., si se reconoce la marca entonces se conoce un aspecto del partido político, aunque muy pequeño, pero si no se reconoce la marca prácticamente no existe en la mente de una persona otra opción es que se confunda la marca con otro partido político y eso puede ser peor porque se atribuyen aspectos que no corresponden, que podrían ser malos y perjudicar al partido 
político en este caso el partido político PDC tiene ambos problemas.

Por lo tanto, el nivel de posicionamiento del partido político PDC se debe al conjunto de todas las características ya mencionadas. Por otra parte, en el mercado político es muy difícil de agradar y no se debe pensar solo en el diagnóstico del posicionamiento actual si no en el crecimiento constante a través del transcurrir del tiempo.

Es de gran importancia terminar con la problemática identificada, el posicionamiento, puesto que, si no se lo resuelve, el partido político "PDC" (Partido Demócrata Cristiano) tiene una gran probabilidad de perecer en el municipio de Potosí.

Al tener conocimiento sobre la problemática, se decidió realizar una PRUEBA PILOTO para cerciorarse $y$ fundamentarse mediante información netamente actual verídica y primaria, mediantes el desarrollo de tácticas de ciberpolítica para incrementar el posicionamiento del partido político "PDC" (Partido Demócrata Cristiano) en la ciudad de Potosí."

La importancia que tiene este estudio en el área, ya sea administrativa o comercial es la innovación en temas de investigación no vistas antes, que en un futuro apoyarán como base a generaciones venideras de estudiantes, instituciones, agrupaciones sociales, partidos políticos y otros interesados.

\section{MÉTODO}

Para el desarrollo de la investigación se realizó mediante el enfoque mixto cuantitativo-cualitativo. Cuantitativo en razón de que establece un pilar primordial para la investigación que es la recolección, análisis e interpretación de datos, obtenida de una muestra determinada, en una prueba piloto donde se cercioró y fundamentó la información actual verídica y primaria, es por esta razón que se llevó a cabo una encuesta en la Universidad Privada Domingo Savio, en la cual se realizaron 50 encuestas, a personas entre 18 a 70 años, con preguntas de opción múltiple (se dan opciones de respuesta y se pide seleccionar una o más de las alternativas dadas), el proceso de tabulación e interpretación se desarrolló en la prueba piloto. Otro enfoque a utilizar fue cualitativo, con el resultado de este enfoque se podo determinar e interpretar de mejor manera los datos de la investigación principalmente en la variable dependiente. Además el tipo de investigación que se utilizó fue descriptivo al tener entendido, es decir que se describió en un tiempo actual y detallado las dos variables identificadas: una a desarrollarse que sería tácticas de ciberpolítica y la otra el posicionamiento del partido "PDC" (Partido Demócrata Cristiano) en la ciudad de Potosí.

Por otra parte, mediante el diseño de Investigación, se estableció una estrategia determinada y orientada hacia la obtención de información principalmente referidos a la de las variables de investigación. Es por esto, que se tomaron en cuenta los aspectos de recopilación documental y trabajo de campo.

\section{RESULTADOS Y DISCUSIÓN}

\section{Etapa diagnóstica}

\section{En la observación}

Otra herramienta que se utilizó, para el desarrollo del diagnóstico, fue la observación, la cual se basó en una guía de observación y se generó los siguientes resultados: La imagen utilizada actualmente, es regular tomando en cuenta las 
características (Ilamativa y agradable), Si toma en cuenta las necesidades de los ciudadanos, ya que su tendencia es principalmente el contacto con la población y el servicio a la sociedad, de forma regular cuenta con una responsabilidad social pero no se puede aún afirmar que también exista una responsabilidad económica.

Las propuestas que presenta el partido político PDC son personalizadas, van a cada sector de la sociedad, se demuestra compromiso con estas propuestas y programas que tiene. Un aspecto en contra, que se pudo observar es que el partido PDC no maneja las herramientas digitales actuales, por lo tanto, no desarrolla una comunicación masiva, es decir no utiliza BTL.

Esta situación se confirma al observar, que no cuenta con una página de Facebook, de WhatsApp, Instagram y Canal de YouTube netamente municipal. La base de un plan adecuado para un mayor posicionamiento es la organización mediante un cronograma de actividades, el cual tampoco se observó en el Partido Demócrata Cristiano.

De la misma manera se observó, que no existe una cantidad determinada, destinada para el uso de publicidad clásica o moderna solo se cuenta con aproximados y no con un presupuesto adecuado.

\section{De acuerdo a la entrevista}

Dentro del trabajo de Campo se realizaron dos entrevistas con una guía de entrevista, basada en la operacionalización de ambas variables.

De acuerdo al resultado de la entrevista realizada, al Secretario General Departamental del partido político PDC la ciudad de Potosí: Lic. J. Miguel Sanabria Solíz, Respecto a los partidos políticos actualmente: ...el mercado político es muy complejo existen partidos políticos que son sobrevivientes a las crisis políticas del país, inclusive muchos de los partidos políticos son enclaustrados de alguna forma por los partidos en función de gobierno que han pasado por la presidencia de Bolivia, esto genera que distintos partidos desaparezcan. Cuando se habla del PDC (Partido Demócrata Cristiano) se observa el mismo panorama, la experiencia por los años que tiene este partido le han permitido continuar en la lucha por conseguir un lugar en la mente de las personas....

Respecto a la imagen: "La imagen de un partido político es caracterizada por la marca, es un aspecto de vital importancia en el caso del partido PDC la marca actual no es muy aceptada, a comparación de otras opciones que también identifican al partido con la diferencia que en estas otras marcas sí se lo puede identificar con las siglas".

Por otro lado, el color que identifica al partido político PDC es del agrado de los ciudadanos, por debajo del color que identifica al municipio de Potosí, pero a pesar de agradar la combinación de colores que se tiene por parte del PDC, no identifica al partido, no se puede hacer referencia al partido PDC, cuando se observa en determinada situación esta combinación de colores y en muchos casos genera confusión al no saber a qué partido hace referencia la combinación de los tres colores que se tienen.

Respecto a la competencia, se realizó una entrevista al Funcionario del Órgano Judicial, Abg. Edwin Aguayo Arando, con el fin de conocer que perspectiva $y$ características principales se utilizan en un partido político para tener un 
posicionamiento en el mercado político actual.

Con respecto a los partidos políticos, indica que "existe varios partidos políticos que en su mayoría tratan de adecuarse a una nueva tendencia, pero el punto en contra de estos es la presencia y representación de políticos que con el transcurrir del tiempo siguen siendo los mismos".

Con respecto a las necesidades: "Una característica importante, que hace que un partido político sea aceptado y tenga posicionamiento, es saber identificar las necesidades principalmente sociales que son vulneradas, para tener una lectura adecuada de estas necesidades, juegan un papel muy importante los actores políticos de cada uno de los partidos dentro del mercado político actual".

Respecto a la inversión: "En el tema de la inversión, no existe una norma de financiamiento para las campañas políticas, en algunas ocasiones existe el autofinanciamiento, pero esto no siempre define la calidad de un partido político".

Respecto al objetivo: "Por otro lado, el objetivo de un partido político con posicionamiento es proponer un proyecto de país, basándose en una política de gobierno, con responsabilidad social y económica que llegue a toda la población y ayude a salir del letargo que tiene el país en comparación con otros países".

Con respecto al candidato: "Cuando se habla de la representación de un partido político, específicamente del candidato en el contexto actual, este debe ser visionario, con mucho sentido común y carisma. El partido político puede ayudar en el desempeño de su candidato con códigos de conducta, pero un candidato debe ser ético sin importar el tipo de códigos que se le impongan".

La percepción e intuición de un candidato puede generar y mejorar las cualidades y aptitudes de este, que ayuden en una mejor representación, siempre y cuando el partido político elija de manera adecuada a su candidato.

Respecto a la tendencia: “En la actualidad ya no se demuestra de manera tan rígida las tendencias de derecha e izquierda, podría decirse que estas tendencias ya quedaron obsoletas y se apunta a una tendencia más práctica que beneficia de muchas maneras al desarrollo de un país, departamento o municipio, esta tendencia es el manejo de una economía mixta".

\section{Cierre de acuerdo a las características del diagnostico}

Después de haber puesto en práctica las herramientas de entrevista, encuesta y observación, de manera específica se determinó que:

- Imagen. - Según el resultado obtenido de la encuesta, la entrevista y observación, se puede evidenciar de manera clara que no existe un conocimiento del partido político PDC (Partido Demócrata Cristiano), puede ser principalmente por que la imagen utilizada actualmente no es agradable, a comparación de otras que también tienen las características del partido PDC, la combinación de sus colores es aceptada, pero aun así no existe un posicionamiento.

- Mercado. - Utilizando las herramientas de entrevista, encuesta y observación se pudo notar que, dentro del mercado político existen varios partidos políticos como opción, pero la caracterización que agrada a la población es más simple de lo que se cree $y$ aunque el partido PDC (Partido Demócrata Cristiano) cuenta estas características, tampoco genera un 
alto posicionamiento en el mercado político.

- Competencia. - La perspectiva de la competencia va relacionada de una manera similar a la del partido político PDC, pero está siendo aplicada de una forma diferente esto genera que se tenga mayor posicionamiento por parte principalmente tres partidos políticos: MAS (Movimiento Al Socialismo), FRI (Frente Revolucionario de Izquierda) y UN (Unidad Nacional), aunque se tiene más competidores estos tres son los que cuentan con mayor posicionamiento, esta conclusión fue obtenida tomando en cuenta elementos y características obtenidas a través de las herramientas de la observación, entrevista y encuesta.

- Votantes (clientes externos). - De la misma manera al utilizarlas herramientas de observación, encuesta y entrevista, se determina que, en el contexto actual, la población tiene bien definido el tipo de partido político que les agrada, con características basadas en aspectos internos de un partido político, pero también toman en cuenta la forma en la que se relaciona y de alguna manera cumpla con la sociedad, al no conocer bien el partido político PDC (Partido Demócrata Cristiano) las personas no pueden hacer una comparación con lo que les agrada, es por esto que no crean un lugar en su mente para este partido político.

\section{Estructura de las tácticas de ciberpolítica}

Basado en el marco teórico empleado para las tácticas de ciberpolítica, se realiza la siguiente estructura: 


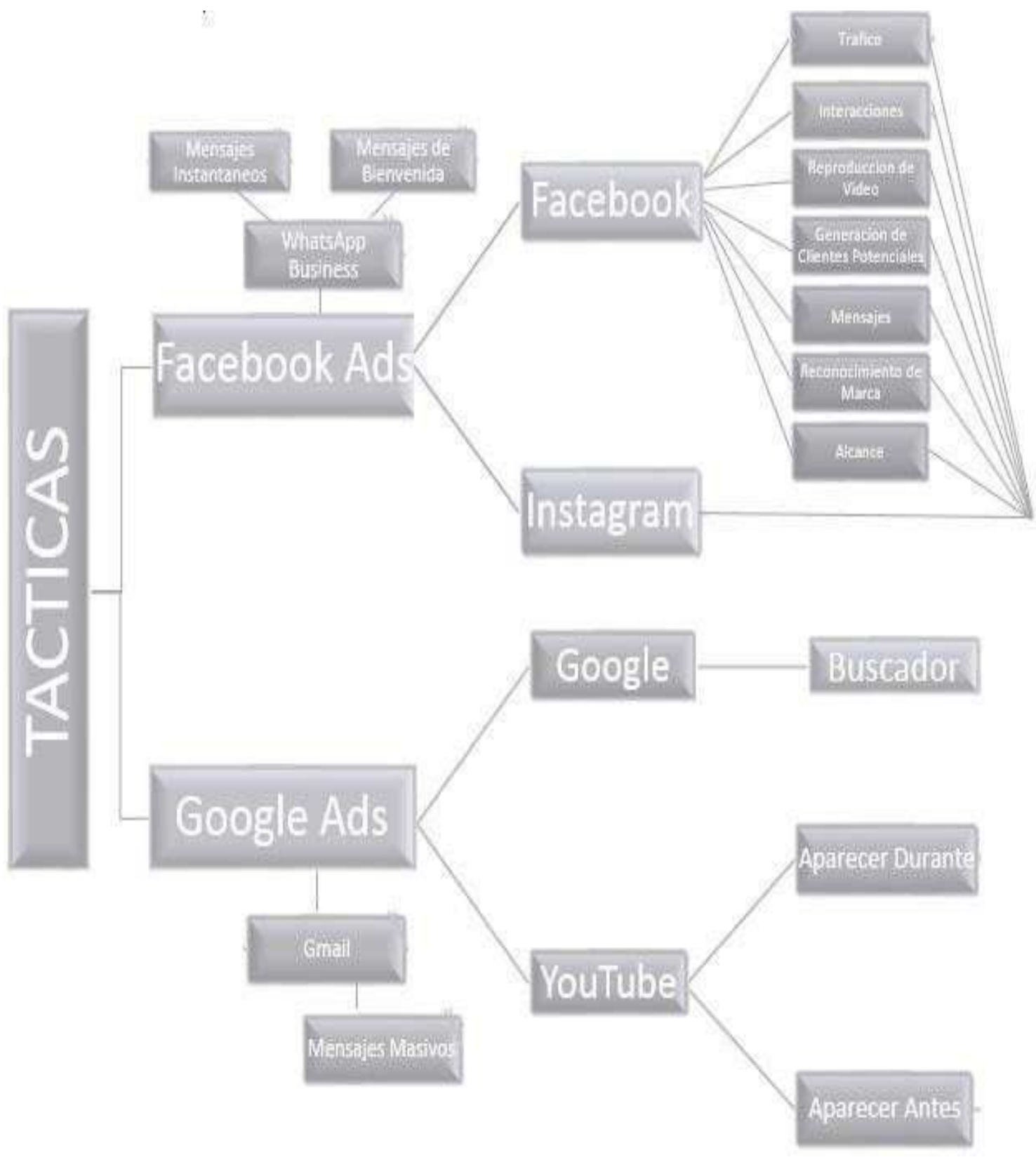

Figura 1. Estructura de las tácticas de ciberpolítica (Elaboración propia) 


\section{Tácticas de Facebook Ads}

Para el desarrollo de las tácticas en Facebook Ads se debe contar con dos principales requisitos:

\section{1.- "Creación de una FANPAGE"}

Implica las siguientes acciones:

- Crear una cuenta de Facebook Personal que maneje la FANPAGE

- Creación de la fANPAGE determinando el rubro al que pertenece:

- Selección del nombre y la categoría de la empresa (Partido Político)

- Conclusión de la elaboración de la FANPAGE.

- Identificación del partido mediante la Imagen. La foto de PERFIL fue seleccionada de acuerdo al agrado que mostraron las personas encuestadas. La foto de PORTADA se obtuvo de acuerdo al agrado que mostraron las personas encuestadas y son los colores del partido PDC

\section{2.- Vinculación de tarjeta de débito}

Se debe realizar la vinculación de la tarjeta de débito, para poder anunciar (promocionar) a la empresa, puesto que si no se paga no existe la forma de llegar a más personas y posicionar el partido político en Facebook.

No es recomendable utilizar una tarjeta de crédito, ya que implicaría mayores costes en el interés y mantenimiento de la tarjeta, es por esta razón que se sugiere utilizar una tarjeta de débito de Banco Unión o Banco Nacional de Bolivia.

\section{Tráfico}

Dentro de esta táctica se tiene como objetivo, dirigir a las personas a ponerse en contacto con el partido político PDC, mediante Messenger y llevar a cabo esta táctica se realizarán las siguientes acciones:

- Se estableció el nombre de la campaña y presupuesto a utilizarse.

- El presupuesto a utilizarse en esta campaña es de $600 \mathrm{Bs} / \mathrm{m}$ ya que se establece la utilización de 20Bs por día en el cronograma realizado, con esta cantidad se podrá alcanzar de manera efectiva a un promedio de 133.500 personas al mes para lograr un mayor posicionamiento.

- Se deberá seleccionar a donde se dirigirán las personas (trafico) cuando se interesen en el anuncio en este caso se seleccionó Messenger. $\mathrm{Y}$ porque se utilizará Messenger, la razón es porque se cuenta con las opciones adecuadas para que el partido político PDC, este en permanente contacto con las personas a las que se puede llegar con la presente táctica.

- Se realiza la segmentación demográfica y especial. Tomando en cuenta el rango de edad determinado en la población este es de 14 a 70 años, la razón de utilizar este rango fue determinado en la población, en la segmentación especial se toma en cuenta intereses que puedan acercar a las personas al partido político, en la táctica se utilizaron intereses como la política, cambio social, personas solteras, casadas, y de otros estados civiles, profesionales, estudiantes y otros.

- Se debe definir las fechas de realización de la campaña, la definición de las fechas se determinará en base al cronograma elaborado y al presupuesto determinado en la investigación, tomando en cuenta siempre el alcance que es un promedio de 133.500 personas al mes.

- Se confirma la identidad del partido, indicando la página FANPAGE del partido 
político PDC. Además de enlazar con la cuenta Instagram de PDC, que contribuirá a la realización de tácticas de Instagram descritas más adelante.

- Se estableció el formato de en el que se verá el anuncio, de acuerdo a la campaña que se lanzará. Esta decisión se tomará de acuerdo al Arte (Imagen o video) con el que se cuente para el anuncio, ya que se tiene las opciones de anuncios por secuencia, una sola imagen un solo video y presentación, en la presente táctica se seleccionó la opción de una sola imagen, puesto que se presentara solo una imagen (Arte).

- Descripción del Arte a utilizarse. El Arte presentado a continuación fue elaborado por la empresa de diseño gráfico Navia Art. (Empresa Tercerizada).

- Se seleccionó la imagen para el anuncio. También llamado Arte, es la representación gráfica de lo que se querrá mostrar ante las personas, Para esta campaña se tomó en cuenta un atributo que resalta como favorito (JUSTICIA), en la encuesta realizada.

- Se realiza la configuración para el anuncio, redactando el texto que será la parte descriptiva de la imagen, en este se indica de manera específica todo lo que se desea hacer conocer en la campaña. Este contenido está basado en la JUSTICIA un atributo obtenido mediante la encuesta, en este contenido se desarrolla una leyenda, basada en un autor que es Paul Aster e indica que la justicia es para toda la sociedad y cuando deja de ser así ya no esjusticia.

- Como el tráfico va dirigido hacia Messenger, se realiza la configuración en la parte final, para que todas las personas al pedir "más información", se deriven a una comunicación directa por Messenger.
Esta táctica coadyuvara en incrementar el posicionamiento del partido político PDC, teniendo en cuenta el alcance que es un promedio de 133.500 personas al mes y mediante el cual se estará en contacto directo con las personas a las que se llegue con esta táctica.

\section{Interacción}

Dentro de esta táctica se quiere llegar a poseer interacciones ya sea dé "me gusta" o "participación en eventos", que el partido PDC anuncie, tendrá las siguientes acciones:

- Al trabajar la campaña con la publicación o la página con un evento, también se define el nombre de la campaña y el presupuesto.

El presupuesto a utilizarse en esta campaña es de 600Bs. al mes ya que se establece la utilización de 20Bs por día en el cronograma realizado, con esta cantidad se podrá alcanzar de manera efectiva a una cantidad considerable de personas para lograr un mayor posicionamiento. (Como el presupuesto será el mismo para la táctica, se prevé que se tendrá el mismo alcance que es un promedio de 133.500 personas al mes).

- Las características de segmentación, tiempo, ubicación e identificación permanecen iguales a la de la campaña de tráfico.

- Dentro de la campaña de interacción se debe seleccionar una publicación ya existente de la página.

- Descripción del Arte a utilizarse. El Arte presentado a continuación fue elaborado por la empresa de diseño gráfico Navia Art. (Empresa tercerizada). Se tomó en cuenta la base de Democracia por una de las propuestas principales que ofrece el partido político PDC (Partido Demócrata Cristiano). 
Esta táctica ayudara a incrementar el posicionamiento del partido político PDC, teniendo en cuenta el alcance de las personas que realizaran una interacción con las publicaciones y los contenidos, en un promedio de 133.500 personas al mes.

\section{Reproducción de video}

En la presente tacitica también se tienen un conjunto de actividades a continuación desarrolladas:

- Se estableció el nombre y el presupuesto para la campaña.

El presupuesto a utilizarse en esta campaña es de 600Bs. al mes ya que se establece la utilización de 20Bs por día en el cronograma realizado, con esta cantidad se podrá alcanzar de manera efectiva a una cantidad considerable de personas para lograr un mayor posicionamiento. (Como el presupuesto será el mismo para la táctica, también se tendrá el mismo alcance que es de un promedio de 133.500 personas al mes).

- La identificación, la segmentación, ubicación y tiempo se desarrollarán de la misma forma que en las anteriores campañas.

- Se determinó el formato del video de la campaña priorizando en todo momento el tipo de campaña que se realice, de acuerdo al Arte que será un video o una presentación solo de imágenes.

- Posteriormente se elige el video, o puede crearse con una cantidad determinada de imágenes, todo dependerá la campaña y el enfoque que se quieremostrar.

- El video a ser difundido, contempla principalmente la idea general, que es el cambio referido en distintos aspectos que se tomaron en cuenta en la encuesta realizada se optó por un representante de la población potosina que cuente con las características del potosino promedio, al mismo tiempo se realizó el video en la ciudad de Potosí.

- Seguido de la selección del video se desarrolló el texto dentro del anuncio que describa lo que se busca transmitir con el mismo, en este caso hace referencia al partido político como parte del municipio e incluso siendo en su totalidad el municipio como tal.

El aporte que realizara esta táctica, es mediante la reproducción de video por un promedio de 133.500 personas al mes, por ende, se generara un incremento en el posicionamiento del partido político PDC.

\section{Generación de clientes potenciales}

Dentro la táctica de generación de clientes potenciales se busca obtener más datos de las personas a las que se llega, como el número de teléfono y correo electrónico, se tendrán distintas acciones, las cuales se desarrollarán a continuación:

- Se establece el nombre y el presupuesto de la campaña.

El presupuesto a utilizarse en esta campaña es de 600Bs. al mes ya que se establece la utilización de 20Bs por día en

el cronograma realizado, con esta cantidad se podrá alcanzar de manera efectiva a una cantidad considerable de personas para lograr un mayor posicionamiento. (Como el presupuesto será el mismo para la táctica, por lo cual se tendrá el mismo alcance que es de un promedio de 133.500 personas al mes).

- Al igual que en las anteriores campañas se tomarán en cuenta las mismas características de la segmentación, identificación, ubicación y tiempo.

- Se determinó la imagen para la campaña, teniendo en cuenta que identifique o 
represente de manera correcta lo que se quiere hacer conocer.

En una mayor parte las imágenes (Arte) que se publicaran no contienen mucho texto, esto es porque las normas de Facebook nos indican que a menor cantidad de letras mayor es el alcance que se obtiene.

Posteriormente se desarrolló el llenado del texto dentro el anuncio, como en las anteriores campañas aquí también se debe describir el mensaje, la propuesta o la información que desea transmitirse, en este caso se utilizó netamente la propuesta del partido político, para relacionarlo con la necesidad que tiene la población y es Salud de Calidad.

Como última acción se desarrolló el formulario para la generación de Clientes Potenciales, de este formulario se podrá obtener una base de datos, que permitirá al partido político PDC conocer datos que coadyuvaran en la relación directa con las personas.

La táctica realizada ayudara en la obtención de datos, para tener una relación directa con las personas, incrementando el posicionamiento en un promedio de 133.500 personas al mes.

\section{Mensajes}

La elaboración de esta táctica, permitirá que las personas puedan ponerse en contacto con el partido político PDC mediante Messenger o WhatsApp, cuenta con las siguientes acciones:

- La determinación del nombre y presupuesto para la campaña

El presupuesto a utilizarse en esta campaña es de 900Bs. al mes ya que se establece la utilización de 30Bs por día en el cronograma realizado, con esta cantidad se podrá alcanzar de manera efectiva a una cantidad considerable de personas para lograr un mayor posicionamiento. (Como el presupuesto será mayor en comparación con las demás tácticas, se tendrá un promedio de 196.500 personas al mes).

- Elegir el lugar de destino de los mensajes en este caso será WhatsApp Business, una plataforma de mensajes que le permitirá al partido político PDC, estar en constante comunicación con las personas.

- Las acciones de segmentación, ubicación y tiempo serán las mismas de las anteriores campañas ya desarrolladas.

- Se procede a la selección de imágenes para el anuncio durante la campaña.

- Como última acción, se tiene la selección de enlaces y llenado de texto para la campaña, teniendo en cuenta que se realiza la vinculación directa de la cuenta de WhatsApp Business.

Esta táctica mediante la vinculación con WhatsApp Business, que permitirá estar en contacto permanente con las personas, generará un incremento de posicionamiento al llegar a un promedio de 196.500 personas al mes.

\section{Reconocimiento de marca}

Dentro de la cual se desea generar el reconocimiento de la marca, ante personas que puedan interesarse en ella, se deben realizar las siguientes acciones:

- Se determinará el nombre de la campaña, y el presupuesto.

El presupuesto a utilizarse en esta campaña es de 600Bs. al mes ya que se establece la utilización de 20Bs por día en el cronograma realizado, con esta cantidad se podrá alcanzar de manera efectiva a una cantidad considerable de personas para lograr un mayor 
posicionamiento. (Como el presupuesto será similar al de otras tácticas, se tendrá el mismo alcance que es de un promedio de 133.500 personas al mes).

- Se determinó la región donde se realizará la campaña de la publicación, también llamada segmentación geográfica, en este caso se utilizó el municipio de Potosí.

- Se realiza una segmentación demográfica, tomando en cuenta la edad, sexo e idioma, siempre bajo las características que se determinaron en la muestra.

- Se realiza una segmentación detallada, que incluye intereses y comportamiento de las personas en Facebook, como la política, cambio social, personas solteras, casadas, y de otros estados civiles, profesionales, estudiantes y otros.

- Se determinó la hora y fecha, aquí se puede elegir el día que comience y el día que termine la campaña creada, incluso de que hora a qué hora se desea establecer el inicio y el final.

- Desarrollo de la identidad, como estará representado el partido político, en esta etapa se deberá vincular la cuenta de Instagram, para poder realizar la misma táctica, en esa herramienta digital, desarrollada más adelante.

- Configuración de la imagen para el anuncio, en este caso se tomó en cuenta dentro del Arte en una esquina la combinación de la bandera en de rojo y blanco, que fue la que más agrado a las personas que respondieron la encuesta.

- Edición del Texto para el anuncio, además de la redacción del texto se deberá colocar la vinculación con la página web del partido político, para que se pueda otorgar más información acercan del partido político PDC a las personas a las que se llegue.
Esta táctica coadyuvara, en llegar a las personas que posiblemente estén más interesadas en conocer el partido político PDC, siendo un promedio de 133.500 personas al mes, que también generara un incremento en el posicionamiento.

\section{Alcance}

Dentro de esta táctica se busca llegar a una mayor cantidad de personas, consideraron las acciones a continuación:

- Establecer el nombre y presupuesto de la campaña

El presupuesto a utilizarse en esta campaña es de 600Bs. al mes ya que se establece la utilización de 20Bs por día en el cronograma realizado, con esta cantidad se podrá alcanzar de manera efectiva a una cantidad considerable de personas para lograr un mayor posicionamiento. (Como el presupuesto será el mismo para la táctica se tendrá, de alcance un promedio de 133.500 personas al mes).

- Dentro de las acciones posteriores se tomará de igual manera las características de las anteriores tácticas, refiriéndose a la segmentación, ubicación, formato e identificación así mismo se escogerá una imagen ya utilizada en anteriores acciones, llegando a la parte principal que hace la diferenciación a estatáctica.

- Desarrollo de texto y definición de destino del alcance, como se pudo observar en las campañas anteriores aquí también se redacta un texto que describa lo que se desea transmitir con la imagen, en este contenido se tiene una idea general del partido político como tal, mostrándose como la mejor opción en el municipio y haciendo énfasis en la responsabilidad y democracia. 
Esta táctica ayudara a llegar a las personas en mayor cantidad, en este caso de acuerdo a la inversión, se llegará a un promedio de 133.500 personas al mes o más, que incrementara el posicionamiento del partido político PDC.

\section{Tácticas de Facebook Ads (Instagram)}

Dentro del manejo de Facebook Ads se pudo evidenciar, que existe, el enlace para la combinación de las tácticas de Facebook con Instagram, para esto se debe contar con una cuenta en Instagram (La foto de perfil de la cuenta es la misma que se utiliza en la página de Facebook y bajo la misma base siendo este el agrado de las personas.

Es por esta razón, que el desarrollo de acciones ya realizadas, son las tácticas también para Instagram (Exceptuando una), siendo estas:

- Trafico

- Interacción

- Reproducción de video

- Mensajes

- Reconocimiento de marca

- Alcance

\section{Tácticas de WhatsApp Business}

Para la realización de las Tácticas de WhatsApp Business, se depende exclusivamente de la base de datos que se obtendrá de la táctica de Generación de Clientes Potenciales y de Mensajes de Facebook Ads.

Para realizar las tácticas se debe contar con una cuenta en WhatsApp Business, que identifique al partido político con su marca.

\section{Mensajes de bienvenida}

Se elabora la redacción del mensaje de Bienvenida, para las personas que accederán a esta cuenta, este mensaje debe contener el
Nombre del partido para generar familiaridad y cortesía.

\section{Respuesta rápida}

De la misma forma, se realizará mensaje de respuesta rápida para todos los usuarios que se contacten con el partido Político PDC buscando más información:

Estas tácticas permitirán al partido político PDC, estar en constante comunicación directa con las personas que accedan al WhatsApp del partido, ya sea para pedir información, dar sugerencias, realizar algún comentario y otros, de alguna manera estrechara los lasos de cercanía con la población y generara un conocimiento del partido por parte de las personas e incrementara el posicionamiento.

\section{Tácticas de Google adwords}

\section{Pertenecer a google adwords}

Se debe tomar en cuenta, que para realizar las tácticas se debe contar con una cuenta en Google AdWords, y también se debe vincular la tarjeta de débito como en Facebook ads.

\section{Creación de campaña en google adwords (buscador)}

Esta táctica se emplea para que, al momento de ingresar en el buscador una opción, la primera que se vea sea la del partido político PDC, esto implicara las siguientes acciones:

- Establecer inversión, de acuerdo al alcance que se espera llegar, esta inversión será establecida por día, mes, año:

- Selección de Redes de Búsqueda o Red Display, estos incluyen la búsqueda en sitios ajenos a Google que utilizan Google como motor de búsqueda. 
- Establecer palabras claves, se colocan opciones de palabras que sirvan como un enlace, para que aparezca el anuncio del Partido Político PDC.

- Redacción del anuncio, en este paso es necesario redactar todo lo que se quiere mostrar en el anuncio, este deber ser claro y llamar la atención para poder ser posicionado.

Para realizar las tácticas en esta herramienta digital, se debe crear un canal de YOUTUBE.

\section{Creación de la campaña mediante YouTube}

Mediante esta táctica con la utilización de YouTube se pretende llegar a personas que constantemente reproducen videos en esta herramienta digital y consta de las siguientes acciones:

- Determinar el Nombre a utilizarse en la campaña.

- Anuncio del video, este anuncio puede tener la opción de aparecer durante la reproducción de video o anunciarse antes de la reproducción de algún video.

- Selección de donde aparecerá el anuncio, se puede establecer si se quiere que el anuncio solo aparezca en YouTube o también en los socios de video de Google.

- Se debe determinar la ubicación donde se realizará la campaña también conocida como la segmentación geográfica.

- Selección demográfica del público en donde se determinan datos como edad, género, estado parental (pueden elegirse varias opciones o todas).

- Establecer el presupuesto de la campaña, este se determina por día, mes o año.

- Estimación del alcance (semana), la herramienta digital cuenta con un área que nos puede mostrar a cuantas personas se llegara y cuantas veces se mostrara el anuncio con la campaña.

Con las tácticas empleadas en Google Ads, se promedia llegar a un número de 18.500 personas por cada táctica, esto de manera significativa generara un crecimiento en el posicionamiento actual del partido político PDC.

\section{Táctica de Gmail}

Se utilizará una Táctica, enfocada principalmente en Gmail, porque es la cuenta más utilizada en el medio de acuerdo al resultado que se obtuvo en la encuesta realizada en el estudio.

Se depende exclusivamente de la base de datos que se obtendrá de la táctica de Generación de Clientes Potenciales de Facebook Ads.

Para realizar la táctica se debe contar con la cuenta de Gmail.

\section{Mensajes masivos}

Esta táctica se emplea para poder estar en contacto y hacer llegar la marca y aspectos distintos del partido político PDC a las personas. Se realizarán las siguientes acciones:

- Se debe adquirir una base de datos desde Facebook Ads de la táctica de (Generación de Clientes potenciales) y se debe importar a la Hoja de cálculo Excel.

- Se redactará el contenido de los mensajes a enviarse, en este caso se utilizará un mensaje que refleje la Presencia, Preparación Académica y procedencia, que son los aspectos tomados en cuenta en el diagnóstico y permiten desarrollar una propuesta que agrade a las personas. 
- Y como último paso se debe aceptar y enviar los mensajes, el límite de los mensajes gratuitos es de 500 al día, que permitirá llegar en una semana a 3500 personas si se mandaran más de 500 mensajes diarios estos se cobrarían.

Todas estas tácticas, de manera directa o indirecta generaran un mayor posicionamiento tomando en cuenta, que las herramientas digitales escogidas son muy efectivas además de ser las más utilizadas actualmente.

\section{Evaluación de las tácticas de ciberpolítica}

De manera adicional a las propuestas, de las tácticas de ciberpolítica se pudo realizar la evaluación de algunas de estas tácticas, para poder comprobar su efectividad y su funcionamiento.

Se tiene la evaluación principalmente las tácticas de Facebook Ads.

Táctica de reconocimiento de marca se evalúa de acuerdo al alcance que se obtuvo en el transcurso de fechas determinadas que se controla desde el administrador de campañas:

- En resumen, se puede observar, que se obtuvo un alcance de 4479 personas e impresiones de 10583 personas en tan solo 3 días.

Es de esta manera que cada táctica se podrá ir evaluando su resultado: por ejemplo dentro la táctica de "Alcance" se obtuvo un total de alcance de 5230 personas e impresiones de 17343 personas, otra táctica que se evaluó fue la de "Trafico" con un resultado de alcance 4567 personas e impresiones de 12346 personas, de esta manera se irán evaluando las tácticas una a una, cuando se pongan en marcha todas las realizadas en la propuesta.

\section{Discusión}

\section{Marketing}

Se determina que el marketing se utiliza para el beneficio y mejoramiento de cualquier aspecto de la empresa o institución, dependiendo donde se pueda encontrar algún tipo de falla o carencia que no permita cumplir con los estándares de efectividad que debe tener cualquier tipo de institución privada o pública, haciendo principalmente un estudio de las necesidades del mercado al que se busca satisfacer.

Por lo que Tirado, 2012 menciona que el marketing plantea que:

Entendiendo que en la práctica del marketing se sitúa el afán por proporcionar valor y satisfacción a sus mercados. Para ello, es esencial que la empresa sea capaz de identificar las necesidades de su cliente, de diseñar y desarrollar ofertas ajustadas a las mismas y de transmitirlas y acercarlas de forma efectiva hacia su mercado. (p.16).

De acuerdo a Kotler y Armstrong (2007) el marketing es el: "Proceso mediante el cual las compañías crean valor para los clientes y establecen relaciones estrechas con ellos, para recibir a cambio valor de los clientes" (p.4).

Para Kotler (2012): “... el marketing es un proceso social y administrativo mediante el cual grupos e individuos obtienen lo que necesitan y desean a través de generar, ofrecer e intercambiar productos de valor con sus semejantes" (p. 7).

Stanton, Etzel y Walker (2007), definen que: "El marketing es un sistema total de actividades de negocios ideado para planear productos satisfactores de necesidades, asignarles precios, promover y distribuirlos a 
los mercados meta, a fin de lograr los objetivos de la organización" (p. 6).

Para la American Marketing Asociación (A.M.A., 2013), "... marketing es la actividad, conjunto de instituciones y procesos para crear, comunicar, entregar e intercambiar ofertas que tienen valor para clientes, clientes, socios y la sociedad en general"

Entonces se considerará al marketing como un proceso, con el cual se conseguirá obtener un valor determinado, además un acercamiento hacia el mercado al que se busca llegar, mediante distintos tipos de canales.

\section{Posicionamiento}

Son varias percepciones y estudio que se hacen con relación a este punto es por ello que de acuerdo con Lambin (2003) "El Posicionamiento define la manera en que la marca o la empresa desea ser percibida por los compradores objetivos" (p. 219).

Según Staton et al. (2007): “... el posicionamiento es el uso que hace una empresa de todos los elementos de que dispone para crear y mantener en la mente del mercado meta una imagen particular en relación con los productos de la competencia" (p.163).

Otra definición que habla del posicionamiento de acuerdo a Ries y Trout (2002) indica que es: “... la forma de diferenciarse en la mente de su cliente prospecto" (p.3)

Como se podrá evidenciar en las definiciones de posicionamiento existe una característica única y especial, que es la mente del cliente o consumidor la tarea principal del posicionamiento es tener un espacio ahí, utilizando como una eficaz y poderosa herramienta de gran alcance al marketing, con diferentes tipos de estrategias o tácticas determinadas para el mercado meta en el cual el posicionamiento busca un espacio.

El posicionamiento como tal, llegaría a ser el resultado de la acción de un tipo de marketing determinado, de acuerdo al área o sector en el que se utilice y como se lo ponga en práctica.

Después de desarrollar distintas definiciones de posicionamiento, se puede determinar que es un aspecto clave, del cual carece el partido político estudiado es en este entendido que se lo tomara como la variable dependiente.

Existe una relación muy estrecha incluso dependiente entre la segmentación y el posicionamiento, y esta es el marketing el cual forma parte fundamental dentro de sus estrategias de la siguiente manera de acuerdo a Alles (2012):

1. Identificar diversas necesidades y grupos en el mercado (segmentación),

2. Centrarse en las necesidades y grupos que pueda atender mejor (definición del público objetivo)

3. Posicionar su oferta de modo que el mercado objetivo reconozca la oferta y la imagen distintiva de la empresa (posicionamiento). (p.42)

Es entonces como una característica importante y básica para lograr el posicionamiento, se determinará mediante la segmentación, tomando en cuenta aspectos como base por la segmentación geográfica y demográfica.

\section{CONCLUSIONES}

En el estudio, se encaró una serie de aspectos teóricos estrechamente relacionados con el estudio del posicionamiento, por lo cual se concluye que es una característica de gran importancia para el partido político en la actualidad, en 
pocas palabras es la medición del lugar que ocupa en la mente de las personas y el resultado de dicho posicionamiento es muy bajo.

Se establece que el problema principal es el bajo posicionamiento, determinado así, por el resultado de la sistematización de la investigación, principalmente relacionándolo con la competencia, la falta de conocimiento de las propuestas el nombre, el candidato y la marca se llega a la conclusión de que el partido PDC (Partido Demócrata Cristiano), tiene aspectos a favor que no son difundidos y no pueden crear un lugar en la mente de las personas.

Las propuestas de tácticas de ciberpolítica, apuntan a generar el reconocimiento favorable y distinto, frente a la competencia, obteniendo una marca, imagen del candidato, conocimiento de propuestas y distintos atributos atrayentes para las personas. Creando así afinidad de las personas con el partido político PDC (Partido Demócrata Cristiano), con el resultado de incrementar el posicionamiento que tiene actualmente.

La realización de las propuestas, con un promedio de alcance generan una idea elemental del posicionamiento que se podría lograr al ponerlas en práctica es así que se demuestra con dos o tres tácticas ya elaboradas en la evaluación realizada.

\section{REFERENCIAS}

Alles, M. T. (2012). Dirección de Marketing. Recuperado de http://rodin.uca.es/xmlui/bitstream/han dle/10498/14887/libro\%20dir\%20mk\%2 0en\%20espa\%C3\%B1ol.pdf

Kotler, P. y Armastrong, G. (2007). Marketing Versión Para Latinoamérica. (11a.ed). México: PEARSONG EDUCACION.

Kotler, P. (2012). Dirección de Mercadotecnia (8a. ed.).Naucalpan de Juárez, México: PEARSON EDUCACION.

Lambin, J. (2003). Marketing Estratégico. España: ESIC EDITORIAL

Ries, A., Y Trout, J. (2002). Posicionamiento: la batalla por su mente. McGraw-Hill.

Staton, J., Etzel, J. y Walker, J. (2007). Fundamentos de Marketing. (14a..ed).México: McGraw-Hill Interamericana editores, S.A. DE C.V

Tirado, D. (2012). Fundamentos de Marketing Recuperado de: http://repositori.uji.es/xmlui/bitstream/ handle/10234/49394/s74.pdf 